\title{
A Comparison of Minimum Risk Portfolios under the Credit Crunch Crisis
}

\author{
Theodoros Mavralexakis ${ }^{1}$, Konstantinos Kiriakopoulos ${ }^{2,3}$, George Kaimakamis ${ }^{4}$, Alexandros Koulis ${ }^{3}$ \\ ${ }^{1}$ Proton Bank, Risk Management Department, Athens, Greece \\ ${ }^{2}$ Proton Bank, Capital Markets, Athens, Greece \\ ${ }^{3}$ Department of Regional Economic Development, University of Central Greece, Lamia, Greece \\ ${ }^{4}$ Department of Mathematics and Applied Mechanics, Hellenic Army Academy, Athens, Greece \\ E-mail: t.mavralexakis@proton.gr,k_kiriak@otenet.gr,\{gmiamis,koulisal\}@gmail.com \\ Received July 4, 2011; revised July 24, 2011; accepted August 2, 2011
}

\begin{abstract}
In this paper the behaviour of various popular risk portfolios measures used for portfolio construction are compared using data from the recent financial crisis. Results are revealing the way optimal portfolios should be constructed. Despite the conventional wisdom, short selling gives only a marginal improvement to portfolio performance during the crisis period. Optimal semivariance portfolio produces better results than the portfolio constructed with the more advanced expected short fall method. Additional historical information has added to performance up to a point and long dated history seems not to be commensurate with additional benefits. Rebalancing frequency seems to have an optimal point that favours neither overtrading nor the conventional buy and hold strategy.
\end{abstract}

Keywords: Portfolio Optimization, Expected Shortfall, Minimum Variance

\section{Introduction}

The financial market turmoil, which emerged in late 2007, has led to the most severe financial crisis since the Great Depression. The bursting of the housing bubble forced banks to write off several hundred billion dollars in bad loans caused by mortgage delinquencies. At the same time, the stock market capitalization of the major banks declined by more than twice as much. While the overall mortgage losses were large on an absolute scale, they were still relatively modest compared to the $\$ 8$ trillion of US stock market wealth losses between Oc- tober 2007 when the stock market had reached an all- time high and October 2008. Stulz [1] mentions the need for taking into account events of small probability that can lead to such big losses.

This highlights once again the importance of constructing minimum risk portfolios with no implicit normality assumptions on returns. This work attempts to search for a methodology to construct optimal minimum risk portfolios by capitalizing on different risk measures. Various minimum risk portfolio selection measures, using data from the recent financial crisis, are compared. These measures are used ex-ante, and optimal portfolios are constructed. In this way, the preferences of a riskavert investor who wants to choose portfolios ex-ante based on different minimum risk measures are reflected.

The risk measures optimized are variance (variance minimization-VM), the Expected Shortfall (ES) and the semivariance method (SEMI). Results are compared to the results produced by the naive method (NM) and by using various benchmark indices. Although the variance measure ([2]) is criticized for its simplicity since it uses only the first two moments for portfolio selection and implicitly assumes normality of portfolio returns, it remains a well-accepted measure at least for comparison reasons. Modern VAR or ES can be either as a constraint embedded on broader stochastic portfolio maximization problem $([3,4])$ or as an objective function to be minimized ([3,5-7]).

Extensions when the exit time is uncertain can be found in [8]. The lack of subadditivity of the VAR measure ([9-11]) makes the ES measure a better candidate for optimization since it is consistent to risk management objectives for capital allocation, especially within the scope of the Basel II regulation framework. VAR/ES methods has also been extensively used for derivatives portfolio ([12,13]). Gilli and Schummann [14] investi- 
gate the empirical performance of alternative selection criteria in portfolio optimization. Although the results presented there have a great sensitivity to small data perturbations, alternative selection criteria such as the semivariance measure seems to produce superior results to the variance measure. This is the reason semivariance minimization is included in the portfolio selection criteria. The naive method, although has a questionable efficiency ([15]), is used for reasons of comparison since its diversification is guaranteed. This paper is organized as follows: In Section 2 the optimization methodology is presented. Section 3 includes the data used and the software employed. Our main results can be found in Section 4. Conclusions and extensions can be found in Section 5.

\section{Optimization Methodology}

We employ three minimization programs: the variance minimization, the expected short fall minimization and the semivariance minimization. The objective is to find the optimal portfolios that minimize the programs above and to actually test them during the recent crisis. We use the following notation:

$N$ : is the number of portfolio assets.

$T_{1}, T_{2}$ : are the first and the last day respectively in the period under examination.

$T:$ is the number of trading days in the periods $\left[T_{1}\right.$, $\left.T_{2}\right]$.

$t:$ is the first day of portfolio rebalancing.

$H$ : is the number of trading days in the historical windows used for the calculation of portfolio variance, semivariance or expected shortfall, when needed. It must hold $t-T_{1}>H$.

$F$ : is the number of days until rebalancing. Rebalancing takes place at dates $t+j F, j=0, \cdots, L_{t}$, where $L_{t}$ is the integer part of the ratio $\frac{T_{2}-t}{F}$.

$\chi^{j}=\left(\chi_{1}^{j}, \chi_{2}^{j}, \cdots, \chi_{N}^{J}\right)^{T}, j=0, \cdots, L_{t}$ represent vector of the weights in the $\mathrm{N}$ assets at the $\mathrm{j}$ rebalancing period.

In case of short sale restrictions it holds $0 \leq \chi_{i}^{j} \leq 1$. Moreover, $\sum_{i=1}^{N} \chi_{i}^{j}=1$. Let $\chi$ be the set of all possible admissible portfolio vectors.

Let $P_{i}=\left(P_{1}^{i}, P_{2}^{i}, \cdots, P_{N}^{i}\right)^{T}$ be the asset price vector at the $i$ date. We have the usual budget constraint $\chi_{t}^{T} P_{t}=$ $u$ where $u>0$. The minimum variance program is the usual convex minimization program that is performed on the rebalancing periods:

$$
\min \chi_{\mathrm{x}^{J} \in \chi}^{J^{T}} V \chi^{J}
$$

The variance covariance matrix $V$ is calculated using daily data from the historical window prior to the rebalancing date.
Following Uryasev et al. (2002), for the VAR and expected shortfall program with probability $\beta \in[0,1]$ we first define the function $g(\chi)$ for a portfolio vector $\chi \in \mathrm{X}$

$$
g(\chi)=\alpha_{\beta}(\chi)+\frac{1}{(1-\beta) H} \sum_{k=1}^{H}\left[f\left(\chi, y_{k}\right)-a_{\beta}(\chi)\right]^{+}
$$

where $\alpha_{\beta}(\chi)$ is the $\beta$-quantile of the portfolio loss distribution and $f(\chi, y)$ is the negative daily portfolio return with portfolio vector $\mathrm{x}$ and portfolio daily returns $y=\left(y_{1}, \cdots, y_{N}\right)^{T}$. So we have $f(\chi, y)=-\chi^{\mathrm{T}} y$ and $a_{\beta}(\chi)=\min (a \in R, P(\chi) \geq \beta)$ where $P(\chi)$ is the probability of $f(\chi, y)$ not exceeding the $\alpha$ threshold. The daily portfolio returns are calculated from the portfolio vector prices as a log returns.

The VAR and expected shortfall program is

$$
\min _{\chi^{j} \in \mathrm{X}} g\left(\chi^{j}\right)
$$

where the optimization is performed on the rebalancing periods $j=0, \cdots, L_{t}$. This setting of minimizing $g\left(\chi^{j}\right)$ is a problem of convex programming as $g\left(\chi^{j}\right)$ is convex as a function of $\mathrm{x}$ and VAR and therefore differentiable in these variables. If $\hat{\chi}^{j}$ is the optimal portfolio vector at the rebalancing date $j$ then the $\operatorname{VAR}$ is $a_{\beta}\left(\hat{\chi}^{j}\right)$ and the expected shortfall is $g\left(\hat{\chi}^{j}\right)$.

Finally the semivariance optimization program is obtained similarly to the minimum variance optimization but now instead of variance of returns, the partial moment of semivariance ([16]) is used. It is clear from the above that on each rebalancing date three optimization programs are performed and the optimal VM portfolio, the minimum VAR/ES portfolio and the minimum SEMI portfolio are obtained.

\section{Data and Software}

Four different data sets with only risky assets were considered and time series of closing prices spanning from $1 / 11 / 1997$ to $3 / 3 / 2009$ were used. The credit crunch period was from $1 / 11 / 2007$ to $3 / 3 / 2009$. The data sets were composed of big capitalization stocks, listed in the Greek, European and US market and two zero Greek government coupon bonds with maturities of two and ten years. Greek market has been chosen, because it is within the euro area but still has emerging markets characteristics like increased volatility, increased bid-offer spreads and lack of depth compared to the core European markets during periods of crises. Also the Greek bond market is very suitable for this analysis because on the one hand it is very liquid and is quoted on a spread to the German bond market and on the other hand during the recent crisis has been more damaged compared to other European or American markets. Since in the analysis only risky 
assets are considered, Greek bonds used present a risky class albeit of different nature from stocks. The bond maturities used were capturing the most part of the yield curve, and zero coupon were chosen so that the reinvestment coupon problem to be avoided.

Stocks with sufficient data history, which cover at minimum the historical window, were grouped by their national market and formed different data sets. The data sets are the following: 1) 24 different time series of stocks included in FTSE20 and FTSE40 Greek indexes; 2) 24 time series of stocks included in Dax30 Index; 3) 29 time series of stocks included in Dow 30 index and 4) time series of ASE, Dax 30, Dow 30 indexes plus time series of 10 years and 2 years Greek zero coupon bonds.

The formation of the objective function was based on historical distribution and risk measures instead of risk reward ratios (i.e. sharp ratios) were optimized. Keeping the number of assumption to the minimum, rebalancing frequencies, historical windows, risk measures, data groups and short sales indicators were all variables to be optimized during the credit crunch period.

During the credit crunch period rolling- window back-tests with a historical window of length $\mathrm{H}$, and a holding period of length F, were conducted. Overall, the time evolution of 1440 different portfolios by using 4 historical windows, 9 rebalancing periods, 5 risk measures, 4 data groups and 2 short sales on/off indicators were tested.

Each one of the different historical windows considered was of length 1 year, 2 years, 5 years and 10 years and each one of the holding periods was of length 1 day, 1 week, 2 weeks, 1 month, 2 months, 3 months, 4 months, 5 months and 8 months.

Thus, at point in time $t_{1}$, on data from $t_{1}-H$ to $t_{1}-1$, optimization is performed with the resulting portfolio to be held until $t_{2}=t_{1}+F$. At this point, a new optimal portfolio is computed, using data from $t_{2}-H$ until $t_{2}-1$, and the existing portfolio is rebalanced. This new portfolio is then held until $t_{3}=t_{2}+F$ and so on and so forth until the end of the period. So during the walk forward through the data, wealth trajectories are computed optimizing each one of the above variables considered and holding the others fixed. In order to perform the wealth trajectories through time, the initial portfolio was set to contain only cash in amount of 100,000 EUR. No limits were imposed on the individual positions $\chi_{i}$. The optimal values for each of the above variables, formed the selection criteria of the optimal portfolio to be tested against indexing and equal weight strategies.

The software used for the computations was Matlab R2007b. Optimizations were performed using sequential quadratic programming methods (SQP), which transform the constrained optimization problem into an easier subproblem that can then be solved and used as the basis of an iterative process. Fmincon function capitalizes on this method by solving the constrained problem using a sequence of parameterized quadratic programming unconstrained optimizations (more details about fmincon and constraint non linear optimization exist in http://www. mathworks.com).

\section{Results}

\subsection{Risk Measures Performance}

Interesting results were drawn concerning the choice of the optimal risk measure, the optimal rebalancing period, the optimal historical window and the usage or not of short sales. The main result from the comparison of the risk measures was that portfolios constructed by minimizing partial and conditional moments like SEMI and ES performed better than those constructed by simply minimizing variance. Furthermore, when considered confidence levels for the ES methodology, the optimal level was the $95 \%$ and not $90 \%$ or $99 \%$ confidence levels. Table 1 summarizes the findings where for each risk measure the average portfolio value and the average annual percentage change was calculated.

Table 1 shows that for the different risk measures the portfolios performances are very close to each other with that of SEMI being the optimal.

These findings suggest that during high volatility periods, like the credit crunch crisis, the optimal risk measure selection does not play such a crucial role. Next finding was relative to the rebalancing frequency of the portfolios. If a portfolio is never rebalanced, it will gradually drift from its target asset allocation to higherreturn, higher-risk assets. Compared to the target allocation, portfolio's expected return increases, as does its vulnerability to deviations from the return of the target asset allocation. Therefore there is a trade-off between risk of return deviation and expected return deviation. This trade off accounts for the cost of rebalancing, which in this study is set to 30 basis points for buy transactions and 70 basis points for sell transactions. Optimizations with no transaction costs were run and it was found that transaction costs did not significantly influence the re-

Table 1. Risk measures.

\begin{tabular}{ccc}
\hline Risk Measure & Portfolio Value & Change \\
\hline Variance & $71.32 \%$ & $-22.59 \%$ \\
ES99\% & $71.89 \%$ & $-22.12 \%$ \\
ES95\% & $72.55 \%$ & $-21.58 \%$ \\
ES90\% & $72.11 \%$ & $-21.94 \%$ \\
Semivariance & $\mathbf{7 4 . 4 5 \%}$ & $-\mathbf{2 0 . 0 3 \%}$ \\
\hline
\end{tabular}


sults. Nevertheless as the portfolio was rebalanced more frequently than usual (i.e. daily rebalancing), costs became a bigger drag on performance.

Table 2 summarizes the findings, where for each rebalancing period the average portfolio value and the average annual percentage change were calculated.

Table 2 shows that optimal rebalancing period is every three months. Long rebalancing period were expected, given the fact that the data set was coming from a downward-trending market and therefore exhibited high correlation. High correlated assets tend to move together and eliminate the need for frequent rebalancing. Pliska and Suzok [17] mention correlation, volatility and expected return as the asset class characteristics that influence the rebalancing strategy. Further studies also suggest that market environment also plays a role. In trending markets rebalancing frequency should be decreased as opposed to mean reverting markets where portfolio rebalancing should be applied more often.

A third finding was relative to the use of the optimal historical window $\mathrm{H}$. Using the empirical distribution to derive optimal portfolios means that every set of historical P/Ls forms a different set of scenarios. A large set of scenarios with a long sample period produced distributions that better captured the reality including both high and low volatility periods. On the other hand newer information in a sample is more informative than older one and the longer the sample period is, the more periods over which results distorted by past events are unlikely to recur. Therefore there is a tradeoff between a large set of scenarios and newer more relative information which better capture the current situation. This tradeoff is apparent in the findings given the fact that by using the intermediate five year period the best portfolio performances were accomplished. Table $\mathbf{3}$ summarizes the findings where for each historical window the average percentage portfolio value and the average annual percent-

Table 2. Rebalancing frequency.

\begin{tabular}{ccc}
\hline Rebalancing Period & Portfolio Value & Change \\
\hline 1 day & $52.33 \%$ & $-100 \%$ \\
1 week & $72.20 \%$ & $-22.96 \%$ \\
2 weeks & $74.72 \%$ & $-20.49 \%$ \\
1 month & $76.12 \%$ & $-19.20 \%$ \\
2 months & $75,46 \%$ & $-19.47 \%$ \\
3 months & $\mathbf{7 6 . 5 3 \%}$ & $-\mathbf{1 8 . 7 4 \%}$ \\
4 months & $75.41 \%$ & $-19.74 \%$ \\
5 months & $75.37 \%$ & $-19.48 \%$ \\
8 months & $70.58 \%$ & $-23.54 \%$ \\
\hline
\end{tabular}

Table 3. Historical window.

\begin{tabular}{ccc}
\hline Historical Window & Portfolio Value & Change \\
\hline 1 year & $73.66 \%$ & $-21.31 \%$ \\
2 years & $73.58 \%$ & $-21.35 \%$ \\
5 years & $\mathbf{7 3 . 7 4 \%}$ & $-\mathbf{2 0 . 8 2 \%}$ \\
10 years & $68.87 \%$ & $-24.94 \%$ \\
\hline
\end{tabular}

age change was calculated.

As for the usage or not of short sales, it was found out that by using short sales there was a marginal improvement in the portfolio's performances. Nevertheless the volatility of the wealth trajectories of portfolios constructed with short sales was a lot bigger than the rest and in some cases these portfolios even resulted losing all their initial value. That's short sales were excluded from the selection criteria that formed the optimal portfolio. Table 4 summarizes the findings.

\subsection{Risk Measures and Benchmarks}

Given the above results, the optimal minimum risk portfolio was constructed without using short sales, with rebalancing frequency of every three months and by using five years of data to minimize the semivariance risk measure. The wealth trajectories of this portfolio are tested versus the wealth trajectories of benchmarks formed from the four different data sets.

The benchmarks used for comparison were for the first 3 data sets (Greek equity market, European equity market and US equity market) the relevant equity market indexes and for all 4 data sets including the global data set, which is not directly comparable with a specific stock market index, the naïve equal weight strategy ([5]). The results detailing the wealth trajectories of the optimal portfolio versus the benchmarks for each data set are presented in Tables 5, 6, 7 and 8 .

From these tables (and the corresponding graphs 1 through 4) it is shown that the wealth trajectories formed by the optimal portfolio clearly outperform the wealth trajectories formed by the benchmarks.

Exception to this was the Greek market, where the performances of the optimal portfolio and the performance of the benchmark index were equal.

\section{Conclusions}

In this paper ex-ante minimum risk measures in various

Table 4. Short sales.

\begin{tabular}{ccc}
\hline Short Sales & Portfolio Value & Change \\
\hline No & $73.66 \%$ & $-21.77 \%$ \\
Yes & $73.58 \%$ & $-21.53 \%$ \\
\hline
\end{tabular}

Table 5. Greek market.

\begin{tabular}{cccc}
\hline $\begin{array}{c}\text { Rebalancing } \\
\text { Period }\end{array}$ & $\begin{array}{c}\text { Optimal } \\
\text { Portfolio }\end{array}$ & Equal Weight & General index \\
\hline 1 & 100,000 & 100,000 & 100,000 \\
2 & 85064.78 & 76319.97 & 84489.76 \\
3 & 81539.96 & 76265.07 & 79194.03 \\
4 & 64165.71 & 61659.91 & 64529.23 \\
5 & 38767.88 & 28842.23 & 38984.12 \\
6 & 31639.11 & 25055.39 & 33293.54 \\
\hline
\end{tabular}


Table 6. Europe market.

\begin{tabular}{cccc}
\hline $\begin{array}{c}\text { Rebalancing } \\
\text { Period }\end{array}$ & Optimal Portfolio & Equal Weight & DAX30 \\
\hline 1 & 100000 & 100000 & 100000 \\
2 & 92153.45 & 88792.59 & 86179.51 \\
3 & 92346.11 & 91484.52 & 87781.68 \\
4 & 87570.46 & 84704.49 & 82019.79 \\
5 & 87959.28 & 66777.33 & 60336 \\
6 & 80073.42 & 67781.57 & 58496.55 \\
\hline
\end{tabular}

Table 7. US market.

\begin{tabular}{cccc}
\hline $\begin{array}{c}\text { Rebalancing } \\
\text { Period }\end{array}$ & Optimal Portfolio & Equal Weight & DOW30 \\
\hline 1 & 100000 & 100000 & 100000 \\
2 & 92553.60 & 90036.49 & 90214.66 \\
3 & 97774.04 & 94161.37 & 92411.87 \\
4 & 99179.93 & 86102.95 & 82881.67 \\
5 & 85140.55 & 67320.99 & 63047.37 \\
6 & 81532.31 & 65328.67 & 59966.56 \\
\hline
\end{tabular}

Table 8. Global.

\begin{tabular}{ccc}
\hline Rebalancing Period & Optimal Portfolio & Equal Weight \\
\hline 1 & 100000 & 100000 \\
2 & 99644.36 & 92279.67 \\
3 & 99759.51 & 92201.97 \\
4 & 99523.01 & 85227.92 \\
5 & 100847.08 & 68399.73 \\
6 & 102348.14 & 65903.66 \\
\hline
\end{tabular}

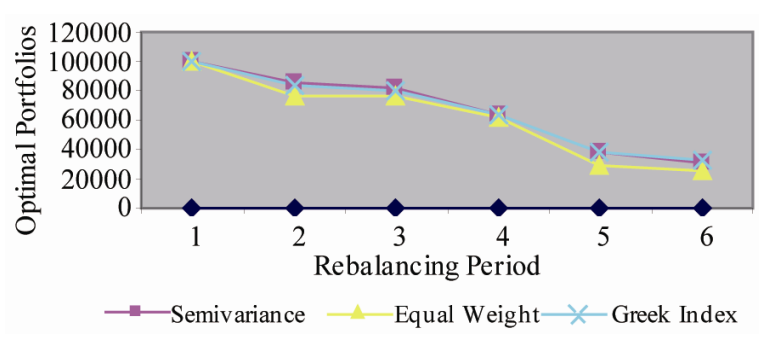

Figure 1. Greek market.

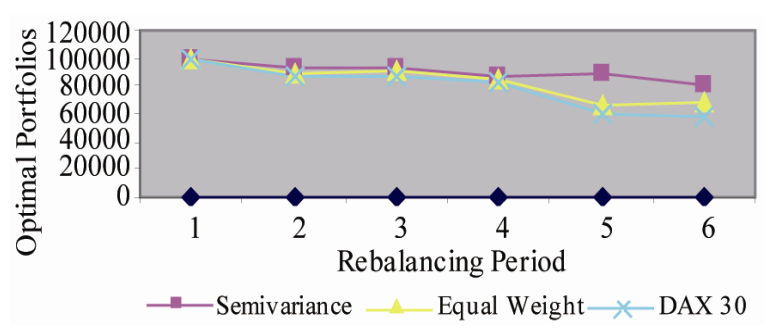

Figure 2. German market.

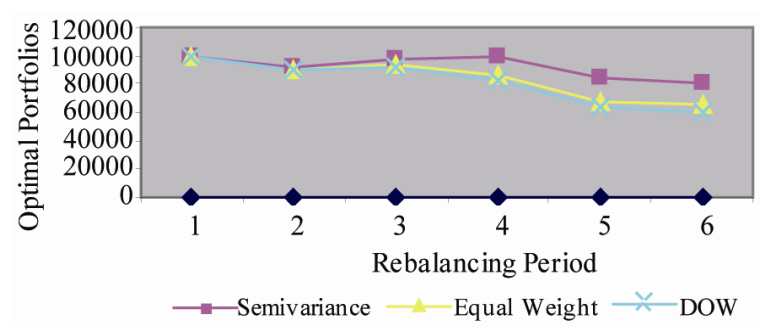

Figure 3. US market.

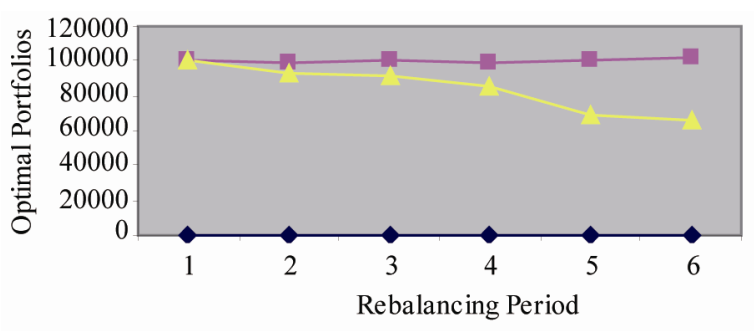

$\because$ - Semivariance $\_$Equal Weight

Figure 4. Global market.

markets during the recent credit crunch crisis were tested. Tests were also performed as far as the optimal rebalancing portfolio period and the optimal historical window are concerned. After the optimal portfolios having being produced, they were compared to the performance of benchmarks indices in each market. The results obtained are more or less the same for all markets. The optimal measure is the semivariance, the optimal rebalancing portfolio rebalancing period is three months and the optimal historical period is five years. The performance of the optimal portfolio clearly outperformed the benchmarks. Inclusion of short sales did not add to the performance even in a crisis period and the inclusion of transaction costs seemed not to influence the performance of the optimal portfolios. Future directions can include derivatives portfolios and the study of risk measures under different volatility regimes (low volatility periods with upward trending or mean-reverting markets). In this way more effective tests could be performed and further insight might be gained concerning correlation among asset classes when structuring the optimal portfolios.

\section{References}

[1] R. M. Stulz, "Risk Management and Derivatives," 1st Edition, Thomson South Western, Florence, 2006.

[2] H. M. Markowitz, "Portfolio Selection," Journal of Finance, Vol. 14, No. 1, 1952, pp. 77-91. doi:10.2307/2975974

[3] A. A. Gaivoronski and G. Pflung, "Finding Optimal Portfolios with Constraints on Value at Risk," Proceedings of the 3rd Stockholm Seminar in Risk Behaviour and Risk Management, Stockholm, 14-16 June 1999.

[4] R. Gandy, "Portfolio Optimization with Risk Constraints," $\mathrm{PhD}$ Thesis, Universitat Ulm, Ulm, 2005.

[5] R. T. Rockafellar and S. Uryasev, "Optimization of Conditional Value at-Risk,” The Journal of Risk, Vol. 2, No. 3, 2000, pp. 21-41.

[6] R. T. Rockafellar and S. Uryasev, "Conditional Value-atRisk for General Loss Distributions,” Journal of Banking and Finance, Vol. 26, No. 7, 2002, pp. 1443-1471. doi:10.1016/S0378-4266(02)00271-6 
[7] P. Krokhmal, J. Palmquist and S. Uryasev, "Portfolio Optimization with Conditional Value-at-Risk Objective and Constraints,” Journal of Risk, Vol. 4, No. 2, 2002, pp. 43-68.

[8] D. Huang, S. Zhu and F. Fabozzi, "Portfolio Selection with Uncertain Exit Times: A Robust CVAR Approach," Journal of Economics Dynamics and Control, Vol. 32, No. 2, 2008, pp. 594-623. doi:10.1016/j.jedc.2007.03.003

[9] P. Artzner, F. Delbaen, J.-M. Eber and D. Heath, "Coherent Measures of Risk," Mathematical Finance, Vol. 9, No. 3, 1999, pp. 203-228. doi:10.1111/1467-9965.00068

[10] S. Cheng, Y. Liu and S. Wang, "Progress in Risk Measurement," Advanced Modelling and Optimization, Vol. 6, No. 1, 2004, pp. 1-20.

[11] H. Follmer and A. Schied, "Convex and Coherent Risk Measures,” In: R. Cont, Ed., Encyclopedia of Quantitative Finance, John Wiley \& Sons, Hoboken, 2010, pp. 355-363.

[12] S. Alexander, T. F. Coleman and Y. Li, "Derivatives Portfolio Hedging Based on CVAR,” In: D. Szegö, Ed., Risk Measures for the 21 Century, Wiley, Hoboken, 2003, pp. 339-363.

[13] S. Alexander, T. F. Coleman and Y. Li, "Minimizing CVAR and VAR for a Portfolio of Derivatives," Journal of Banking and Finance, Vol. 30, No. 2, 2006, pp. 583-605. doi:10.1016/j.jbankfin.2005.04.012

[14] M. Gilli and E. Schumann, "An Empirical Analysis of Alternative Portfolio Selection Criteria,” Swiss Finance Institute Research Paper No. 09-06, March 2009.

[15] V. DeMiguel, L. Garlappi and R. Uppal, “Optimal versus Naive Diversification: How Inefficient Is the 1/N Portfolio Strategy," Review of Financial Studies, Vol. 22, No. 5, 2007, pp. 1915-1953.

[16] C. Mamoghli and S. Daboussi, "Optimisation de Portefeuille Dans le Cadre du Downside Risk,” Portfolio Optimization in a Downside Risk Framework, Working Paper, 19 August 2008.

[17] S. R. Pliska and K. Suzuk, "Optimal Tracking for Asset Allocation with Fixed and Proportional Transaction Costs," Journal of Quantitative Finance, Vol. 4, No. 2, 2004, pp. 233-243. doi:10.1080/14697680400000027 